\title{
Health status and occupational risk factors in Greek small fisheries workers
}

\author{
Elpida Frantzeskou ${ }^{1}$, Olaf Chresten Jensen ${ }^{2}$, Athena Linos ${ }^{1}$ \\ ${ }^{1}$ Department of Hygiene and Epidemiology, Medical School, University of Athens, Greece \\ ${ }^{2}$ Centre of Maritime Health and Society, Institute of Public Health, University of Southern Denmark, Esbjerg, Denmark
}

\begin{abstract}
Background: Fishing is an extremely dangerous occupational activity that predisposes to occupational diseases and accidents. Greece, with about $16,000 \mathrm{~km}$ of coastline and its unique morphological characteristics with small islands and peninsulas, represents a strong proof of its great tradition in the fisheries sector since ancient times. The aim of the study was to examine the health status and the health risk factors present in Greek fishery workers, by exploring their working environment, thus providing a current baseline for documentation of the needs for prevention and health promotion.

Materials and methods: An interviewer-administered questionnaire was distributed in 2013 to a random sample of 172 professional small-scale fishermen of the Evros district in North-Eastern Greece. Results: Eighty-eight per cent worked in coastal fisheries and $73 \%$ were using small fishing vessels, less than $10 \mathrm{~m}$ in length overall. Health disorders included musculoskeletal, cardiovascular and visual problems and to a minor degree by hearing, dermatologic and respiratory problems in all age groups. Seventy per cent had experienced at least one occupational accident. Heavy smoking and daily alcohol consumption was seen among nearly half of the fishermen.

Conclusions: The health effects observed are causally related to the work process exposures on board and to diet, smoking, and lack of exercise. This in turn relates to the specific working conditions, the culture and level of education in small-scale fishing that need to be taken into consideration together in the prevention programmes.
\end{abstract}

(Int Marit Health 2016; 67, 3: 137-143)

Key words: fishermen, health, accident, smoking, alcohol, culture and educational level

\section{INTRODUCTION}

Fishing is an extremely dangerous occupational activity that predisposes to occupational diseases and accidents. As described by Roberts [1], fishermen were 52 times more likely to have a fatal accident at work compared with other British workers. The hazard is probably similar worldwide [2]. Data from those countries that collect accurate accounts show that occupational fatalities in the fishing industries far exceed their national average [3].

Almost one fifth (18\%) of the EU-28's fishing fleet is registered in Greece. On average, however, these Greek vessels are small, with an average size of 4.9 gross tons (much less than the EU-28 average of 19.2 gross tons) and an average engine power of 28.8 kilo- watts in 2014 (compared with an EU-28 average of 75.9 kilowatts) [4].

Internationally, work in the fishing industry has mainly focused on preventing accidents and vessel disasters. Medical conditions, including nutrition-related ones, have received less attention. In Greece there is lack of fishery research studies and the few available studies from other countries like Poland show a higher overall mortality of fishermen from all causes, cardiovascular diseases (CVD) in addition to fatal accidents [5-7]. The pattern of hospitalised cases indicates a need to improve working and living conditions in fishing. For this reason fishermen's Occupational Health Services have been constituted in recent years in different countries, such as Denmark [8]. 
The aim is to assess all the related risk factors affecting Greek fishermen's health and safety, taking into account the special characteristics of Greek fisheries and their effect, with the form either of an occupational disease or accident.

The study explores both the influence of purely occupational characteristics of the production process during the fishing activity by types of fishing gear (nets, long lines, trawls, etc.) and those elements of occupational origin predisposing fishermen professionals to smoking, consumption of alcohol, poor eating habits, insomnia, anxiety, depression, and reduced physical activity. The study attempts to evaluate quantitatively those factors by using appropriate scales in order to record their impact as occupational risk factors for workers in the Greek fisheries sector.

\section{MATERIALS AND METHODS}

This study is based on a pilot study, the first carried out in Greece on health and safety in fisheries, which helped in the proper configuration of the present study's research tool (questionnaire) and represented a source of the first main conclusions regarding the health and safety profile of the workforce in the fisheries sector [9]. Thus, the present study represents the natural continuation of this pilot study and was addressed the entire workforce in the fisheries sector in Evros district. Data collection was taking place during 4 months period of time (August 2013-November 2013). Interviews were taken place at working places or at fishermen's homes, by personal or telephone interviews after an appointment.

The source population consisted of all professional fishermen of Greek Evros region registered in the updated lists of Occupational Fishermen Corporation (according to registries of 2013). As a source benchmarking, the fishermen's data was compared with the health of the general population in the Attica Study [10].

\section{FORMATION OF THE QUESTIONNAIRE}

The questionnaire was original and was based on: a) indicators from the European programme DIRERAF [11] for identifying the most important health and safety indicators in fishing; $b$ ) nutritional questionnaire based on the fitting to the Mediterranean diet model (MedDietScore) [12, 13]; c) demographic information; d) occupational history and features of their type of fishing; e) medical profiles including self-reported CVD, injury experiences, alcohol and smoking history; f) assessment of anxiety, depression, insomnia, physical activity measured by scales.

\section{STATISTICAL ANALYSIS}

Data entry and the statistical analysis were performed using SPSS 19. Besides the uni-variable analysis, cross-table analyses were completed in order to select the possible rel- evant independent variables for the multivariable analysis. Logistic regression analysis was employed to estimate the relative risk of occupational accidents and CVD with adjustment to age, gender and the relevant exposure factors.

\section{MEDICAL CONFIDENTIALITY AND CONSENT}

The study protocol complied with the medical research ethics outlined by the National and Kapodistrian University of Athens. Fishermen were given all necessary information about the aim of the study and they fully agreed with it before the interview started. Their ability to stop the interview and withdraw their consent to participate in the study at any point was absolutely clearly explained to them. All data were processed only by the researcher herself and no other person had access to this material. After the completion of the study, the results were expressed in accordance with the medical ethics and confidentiality rules.

\section{RESULTS}

The study included 161 fishermen, out of a total of 172 ( 5 denied to participate and 6 could not be reached) giving a participation rate of $93 \%$. One hundred and twenty-five (78.2\%) fishermen were occupationally active and $35(21.8 \%)$ were pensioners. The gender distribution in the study population was $80 \%$ males and $20 \%$ females and the average age was 53 years (25-91). Most of the fishermen were married ( $79 \%$ ) (only $0.7 \%$ were divorced) and $40 \%$ had children.

The place of birth was a rural area for $51 \%$, whereas $48 \%$ came from an urban area. Only $1.2 \%$ were born abroad. The main part of the sample $-88 \%$ of the study population - worked in coastal fisheries and for $96 \%$ and $91 \%$ fishing was the main and only occupational activity, respectively. On average they started working as a fisherman at the age of 25 and they had worked in the fisheries for 34 years. Fishing was a full-time job for $91 \%$ and $94 \%$ of them were working in the fisheries during all seasons, 12 months per year with an average of $10.9 \mathrm{~h}$ per day. Some other demographic and occupational characteristics (including the types of vessels) are presented in Table 1.

\section{HEALTH INDICATORS}

Table 2 describes the fishermen's main health characteristics with $64 \%$ of those over 50 years of age being obese with a body mass index of 30 or more. Among those over 50 years of age, $89 \%, 65 \%, 63 \%, 72 \%$ and $72 \%$, respectively, had cardiovascular, dermatological, musculoskeletal and respiratory diseases and hearing problems. In those under 50 the prevalences of all type of diseases were lower. The sample included in the study seems to fit moderately to the pattern of Mediterranean diet (32.7 \pm 5.1 ). 
Table 1. Demographic and occupational characteristics of 161 active Greece fishermen

\begin{tabular}{|c|c|c|c|c|}
\hline Family status & Married: 127 (79\%) & Single: 27 (17\%) & Divorced: 1 (1\%) & Other: 5 (3\%) \\
\hline Children & Yes: 64 (40\%) & No: 97 (60\%) & & \\
\hline Birth location & Urban: 77 (48\%) & Rural: 81 (51\%) & Abroad: 2 (1\%) & \\
\hline Educational level & $0-6$ years: $77(48 \%)$ & $7-9$ years: $(22 \%)$ & $10-12$ years: (21\%) & $\geq 13$ years: (4\%) \\
\hline Type of insurance & Agricultural: 132 (83\%) & Seafarers: 27 (16\%) & Other: $1(1 \%)$ & \\
\hline Length of fishing vessel & $>20$ m: 19 (12\%) & $15-20 \mathrm{~m}: 4(3 \%)$ & 10-14 m: 21 (13\%) & < $10 \mathrm{m:} 117(73 \%)$ \\
\hline Engine power of vessel & $<10$ kW: $9(6 \%)$ & 10-20 kW: $46(29 \%)$ & 10-20 kW: 45 (28\%) & > 50 kW: $60(37 \%)$ \\
\hline Fishing vessel conditions & Satisfied: 120 (76\%) & Unsatisfied: 38 (24\%) & & \\
\hline Type of fisheries & Costal: $88 \%$ & Deep sea: $12 \%$ & & \\
\hline Occupational activity & All seasons: 150 (94\%) & Seasonal: 10 (6\%) & & \\
\hline Retired fisherman & Yes: 35 (22\%) & No: 125 (78\%) & & \\
\hline
\end{tabular}

Table 2. Main health characteristics of the sample of Greek fishermen $(n=160)$

\begin{tabular}{|c|c|c|c|c|c|c|}
\hline & & \multicolumn{2}{|l|}{ Age groups } & \multirow[t]{2}{*}{ Odds ratio } & \multirow[t]{2}{*}{$95 \% \mathrm{Cl}$} & \multirow[t]{2}{*}{$\mathbf{P}$} \\
\hline & & $<50$ & $\geq \mathbf{5 0}$ & & & \\
\hline \multirow[t]{3}{*}{ Body mass index* } & $18.5-24.9$ & $10(17 \%)$ & $13(13 \%)$ & 1.00 & - & \\
\hline & $25-29.9$ & $26(43 \%)$ & $41(42 \%)$ & 1.21 & $0.5-3.2$ & NS \\
\hline & $\geq 30$ & $24(40 \%)$ & $43(44 \%)$ & 1.38 & $0.5-3.6$ & NS \\
\hline \multirow[t]{2}{*}{ Hearing problems } & Yes & $9(15 \%)$ & $23(24 \%)$ & 1.81 & $0.8-4.2$ & NS \\
\hline & No & $51(85 \%)$ & $72(76 \%)$ & & & \\
\hline \multirow[t]{2}{*}{ Dermatologic problems } & Yes & $7(15 \%)$ & $13(24 \%)$ & 1.13 & $0.4-3.0$ & NS \\
\hline & No & $51(88 \%)$ & $84(87 \%)$ & & & \\
\hline \multirow[t]{2}{*}{ Respiratory problems } & Yes & 11(19\%) & $28(29 \%)$ & 1.76 & $0.8-3.8$ & NS \\
\hline & No & 48 (81\%) & 69 (71\%) & & & \\
\hline \multirow[t]{2}{*}{ Musculoskeletal problems } & Yes & $46(78 \%)$ & 77 (80\%) & 14.30 & $6.5-31.7$ & $<0.001$ \\
\hline & No & $13(22 \%)$ & $19(20 \%)$ & & & \\
\hline \multirow[t]{2}{*}{ Vision problems } & Yes & $14(23 \%)$ & 57 (61\%) & 5.10 & $2.4-10.4$ & $<0.001$ \\
\hline & No & $46(77 \%)$ & 37 (39\%) & & & \\
\hline \multirow[t]{2}{*}{ Cardiological problems } & Yes & $14(23 \%)$ & $59(58 \%)$ & 1.75 & $0.7-3.6$ & $<0.001$ \\
\hline & No & 46 (76.7\%) & $42(41.6 \%)$ & & & \\
\hline \multirow{2}{*}{$\begin{array}{l}\text { Belief that work in fishing } \\
\text { affects health negatively }\end{array}$} & Yes & 46 (74\%) & 74 (75\%) & 1.00 & $0.5-0.937$ & NS \\
\hline & No & $16(26 \%)$ & 25 (25\%) & & & \\
\hline
\end{tabular}

*Underweight: $\mathrm{BMI}<18.5$; normal: $\mathrm{BMI}=18.5-24.9$; overweight: $\mathrm{BMI}=25-29.9$; obesity: $\mathrm{BMI} \geq 30$

\section{SMOKING}

The details of smoking are presented in Table 3 with 44\% current smokers, $28 \%$ ex-smokers and $28 \%$ non-smokers. Half of the smokers had 60-200 pack-years of smoking (pack-years are calculated by multiplying the number of packs of cigarettes smoked per day by the number of years the person has smoked; 1 pack year is equal to smoking 1 pack per day for 1 year, or 2 packs per day for 6 months, etc.).

\section{CARDIOVASCULAR DISEASES}

The probability of CVD in Greek fisheries' workforce was related to age, sex, the duration of work in fisheries sector, the fitting to the Mediterranean diet and the level of depression (Table 4). The relative probability of CVD in male fishermen was higher than that in females of the same age. Despite this fact, the adherence to the Mediterranean diet and the absence of high levels 
Table 3. Smoking habit characteristics of the sample of Greek fishermen ( $n=160)$

\begin{tabular}{lll}
\hline Pack years & Smoking years & Smoking at work \\
\hline 1-20: $6(8 \%)$ & $1-10: 1(1 \%)$ & Heavy smoking: $16(10 \%)$ \\
21-40: $18(24 \%)$ & $11-20: 3(4 \%)$ & Too much smoking: $26(16 \%)$ \\
41-60: $12(16 \%)$ & $21-30: 13(17 \%)$ & Moderate smoking: $36(23 \%)$ \\
61-80: $11(15 \%)$ & $31-40: 25(33 \%)$ & Light: $18(11 \%)$ \\
81-100: $10:(13 \%)$ & $41-50: 18(24 \%)$ & Non-smoking: $63(40 \%)$ \\
101-120: $11(15 \%)$ & $51-60: 13(17 \%)$ & \\
121-140: $6(8 \%)$ & $61-70: 3(4 \%)$ & \\
141-160: $1(1 \%)$ & & \\
181-200: $1(1 \%)$ & \\
\hline Smoker: 71 (44\%); Ex-smoker: $45(28 \%) ;$ Non-smoker: $45(28 \%)$ & \\
Periods of non-smoking: Yes: $25(22 \%) ;$ No: 91 (78\%) & \\
Average number of cigarettes per day = 36 & &
\end{tabular}

Table 4. Logistic regression of risk factors for cardiovascular diseases in the sample

\begin{tabular}{llllll} 
& Coefficient (B) & S.E. & Wald & OR & P* \\
\hline Age & -0.121 & 0.046 & 4.987 & 0.93 & $<0.02$ \\
Sex (reference value is male) & -1.932 & 0.698 & 4.29 & 0.19 & $<0.05$ \\
Duration of work in fisheries & 0.021 & 0.017 & 7.941 & 1.31 & $<0.01$ \\
MedDietScore & -1.936 & 0.792 & 4.521 & 0.20 & $<0.01$ \\
Score of depression ZUNG & 0.048 & 0.0213 & 7.932 & 0.98 & $<0.01$ \\
Constant & 4.102 & 1.716 & 4.691 & 28.83 & $<0.05$ \\
\hline -2logLik = 185,332, Nagelkerke R2 $=0.429 ;$ *2-tailed Hosmer and Lemeshow Goodness of fit test: 0.552 & &
\end{tabular}

of depression were causing a protective effect against CVD in fishermen.

\section{ACCIDENTS}

In the sample, $70 \%$ had experienced at least one occupational accident in lifetime. Summarising the findings about occupational accident experience (Table 5), it seems that the most important causes of accidents were an object's hurling (e.g., stone, hook, catch) during the hauling of the fishing gear, the severe fatigue/exhaustion, the entry and exit from the boat, walking along the deck, bad weather, and mishandling during throwing the nets or long lines. Furthermore, the most frequent anatomic locations of injury were the fingers of the hand. The most important causes of accident were a) slipped (42\%) due to slippery or deck full of obstacles; b) trapped by the nets at the time of throwing or hauling them (3\%) and c) other causes (55\%). The most important other causes were an object's flung (e.g. a stone) coming up during hauling the nets $-23 \%$, fatigue $-13 \%$, and bad weather conditions - 9\%
Occupational accidents most frequently happened during processes like a) hauling the nets (39\%); b) throwing the nets (14\%); c) handling the catches (10\%); d) repairing the nets $(0.9 \%)$, and e) other tasks (36\%). Most important of other tasks proved to be a) the entry and exit from the fishing vessel (24\%), b) walking along the deck of the vessel (14\%), c) bad weather conditions (9\%), d) traffic accidents while going to or returning from work (12\%).

Digits of the hands were found to be the most frequent anatomical location of injuries (20\%).

The part of the fishing vessel where injuries happen most frequently was the vessel's bow, and this seems to be causally related with the location of the vessel's pulley for hauling the nets that is usually there in coastal vessels of Greek fisheries.

\section{DISCUSSION}

The study attempts to evaluate quantitatively the most relevant occupational risk factors influencing health and wellbeing by using appropriate scales for workers in a sam- 
Table 5. Causes of accidents in the sample of 161 Greek fishermen
1. An object's flung (e.g. stone) coming up during hauling the fishing tool like nets or longlines
2. Collision with another vessel (due to bad navigation, conflicts with fishing or military vessels of other interests)
3. Bad weather conditions either causing vessel's capsizing or not
4. Poor infrastructure and conditions in the working environment
5. Fatigue during returning home after work
6. Handling of catches (e.g. removing the catches from the fishing tools, e.g. the nets)
7. Repairing the damaged nets due to dolphins, crabs, strong sea currents
8. Extreme musculoskeletal loading
9. Insufficient light
10. Slips due to fish's slime, non proper wearing and shoes, narrow space in the vessel, sharp speed drop of vessel's velocity

11. Mishandling during throwing the nets or longlines

12. Bites by sea fauna during hauling or handling the catches

ple of 161 Greek fishermen who represent the $94 \%$ of the work force of professional fishermen in one Greek district, the so called Evros district of North-Eastern Greece (the district includes Samothrace Island). The study explores both the influence of purely occupational characteristics of the production process during the fishing activity and those elements of occupational origin predisposing fishermen professionals to smoking, consumption of alcohol, poor eating habits, insomnia, anxiety, depression, and reduced physical activity.

It is the strength of the study that $94 \%$ of the approached fishermen wanted to participate and that it was possible to get a relatively large questionnaire completed. The weakness of the study could be the recall bias for the pensioners, although they don't exceed $21.85 \%$ of the sample and the majority of them have never really quitted fishing. By this aspect this study is one of the most comprehensive studies of professional fishermen.

It is also worth to mention that there were no fishermen younger than 25 years of age in the study population. This could be explained by the tendency of Greek coastal fisheries' workforce to quit fishing activity due to economic crisis, fish depletion of the Mediterranean Sea and lack of necessary support by the Greek authorities in order to maintain their traditional family owned fishing enterprises. Thus, it seems younger ages are not encouraged to become professional fishermen in the Evros region of Greece.

The demographic characteristics is typically family-owned small-scale costal fishing in rural areas where the sons in the family start early in fishing without any longer education. The educational level of the sample is low with only primary school for $52 \%$. The small scale fishing vessels under $20 \mathrm{~m}$ are not in all countries subject to statutory survey. The study sample consisted of $80 \%$ males and this is very common as males worldwide dominate the fisheries sector. Despite this fact, the percentage of female fishermen in this sample reached $20 \%$, which is one of the highest in the European Union, representing a strong proof of the family character of Greek fishing enterprises, especially those of coastal type. It is interesting that among the female participants of the study there were women who had the experience of even carrying their newborn babies with them on board for breast feeding or leaving the baby alone for a few hours inside the temporary cage in the Evros River Delta, where they had to stay for a few days in order to obtain a good catch, which gives a new dimension to the issue of safety in fishermen's families.

For the great majority of the fishermen participating in the study, fishing represented their main occupational activity (96\%). For $91 \%$ of the sample it represented their only activity and only for $9 \%$ it is considered as a parttime activity. A significant portion of the sample reported being professionally active (78\%), as it is common among fishermen to continue working in fisheries despite the fact they are pensioners. The average age of starting to work as a fisherman for the sample was 25 years. Fishermen in this sample had been working in fisheries for about 33.7 years on average. Coastal fisheries were the main type of fisheries in this sample (88\%). Most of the fishermen of the study ( $94 \%$ of the sample) worked 12 months per year, with an average of $10.9 \mathrm{~h}$ per day. Also, as far as it concerns the fishing fleet characteristics of thesample, itwas reported that $73 \%$ ofsample'sfishermen work in a fishing vessel of less than $10 \mathrm{~m}$ length, $62 \%$ of the sample work in a fishing vessel of an engine power less than 50 kilowatts and $37 \%$ of the sample's vessels have a horsepower of 20 kilowatts maximum. The ma- 
jority of fishermen (76\%) declared their satisfaction from the working conditions on the fishing vessel where they work and only $24 \%$ of the sample did not consider it as sufficient. Of course one should take into account that most of the study's fishermen are repairing their vessels themselves, thus they may have the tendency to overvalue the quality of the maintenance work they perform.

A prevalence of smokers of $48 \%$ was similar to what was found by Marco Jordan et al. [14]; although, it seems to have been lowered. Overweight and obesity have been clearly described too; $64 \%$ of those over 50 years of age are obese with a body mass index of 30 or more and $61 \%$ of those over 50 years of age are overweight.

Furthermore, focusing on those over 50 years of age, we noted that $89 \%$ had cardiovascular, $65 \%$ dermatological, $63 \%$ musculoskeletal disorders, $72 \%$ respiratory disorder and $71.9 \%$ hearing problems. For those under 50 the prevalence was lower for all types of diseases. As hearing problems were quite common (reported by $21 \%$ of the population surveyed), this could be linked to noise exposures while at work; in this type of vessel, it was found that an average noise level in the engine room was from $95 \mathrm{dbA}$ to $105 \mathrm{dbA}$ [15]. Respiratory problems were reported by on average $72 \%$. Marco Jordan et al. [14] found a prevalence of chronic bronchitis, asthma and smokers to be $18 \%, 10 \%$ and $48 \%$, respectively. Dermatological problems were mainly skin lacerations object to secondary infections, fungal infections of skin and nails, skin aging and skin cancer sun induced.

Fishermen in this sample seem to moderately adhere to the Mediterranean model of nutrition. This result reflects a relatively high adherence of Greek fishermen to the traditional Greek Mediterranean diet if compared with the results of a study performed in almost all habitants of small borderline Greek islands of Aegean Sea (islands like Nisiros, Anafi, Patmos, Thymaina, Kasos, Agathonisi, Leipsoi, Fournoi, Tilos, and a part of Rodos island) which concluded that population of small borderline islands has been urbanised in terms of dietary choices, thus raising the consequences in terms of cardiovascular disorders and depression [16]. Alcohol consumption is found to be a daily habit for the present study's fishermen.

Daily alcohol consumption of at least $300 \mathrm{~mL}$ or more was declared by $34 \%$ of the sample and $19 \%$ reported a consumption of $500 \mathrm{~mL}$ or even more per day, which is a finding in agreement with the international experience about fishermen's alcohol abuse [17].

The health risks factors studied included excessive weight, cardiovascular incidents, and dermatologic, mus- culoskeletal, respiratory, and hearing problems. The occupational health risk factors included alcohol, smoking, insufficient sleep and fatigue.

The probability of CVD in Greek fisheries' workforce is related to age, sex, the duration of work in fisheries sector, the fitting to the Mediterranean diet and the level of depression. The probability of CVD in male fishermen is higher than that in females of the same age. Despite this fact, the adherence to the Mediterranean diet and the absence of high levels of depression are causing a protective effect against CVD in fishermen. This is in correspondence with the Attica study where it has been proved that adherence to the Mediterranean diet seems to mediate the unfavourable effect of depression and anxiety on CVD risk [18].

There are no previous studies on health and safety in the Greek fisheries sector. This study is the continuation of a pilot study which was based on a sample of 100 professional fishermen from areas all over Greece (urban, suburban, rural, island, and mainland regions of the country) [9]. The results of the present study are quite in accordance with the findings of the pilot study drawing a profile of the fisheries' population of the country.

\section{CONCLUSIONS}

Working as a fisherman in the territory of Evros district in North-Eastern Greece is a dangerous occupational activity in terms of the risk of accidents and CVD. The health effects observed are causally related to smoking, alcohol consumption and fatigue, which are all related to the specific working conditions and culture in small-scale fishing. Greek fishermen lack education on occupational health and safety matters and occupational services.

\section{KEY POINTS}

1. Working as a fisherman in the territory of Evros district in North-Eastern Greece increases (after adjustment for age and the exposure to other factors) in a statistically significant manner the likelihood of CVD and occupational accident.

2. The health effects observed are causally related to smoking, alcohol consumption and fatigue, which in turn relate to the specific working conditions and culture in small-scale fishing.

3. Fishermen are lacking education on health and safety matters and occupational medicine services - a conclusion which needs to be taken into consideration by the country's authorities and occupational medicine services for the implementation of prevention programmes with effective measures, carefully adapted to the specific conditions present in Greek fisheries. 


\section{REFERENCES}

1. Roberts SE. Hazardous occupations in Great Britain. Lancet 2002; 36: 543-544.

2. Conway GA. Casting their lot upon the water: commercial fishing safety. Lancet Lond Engl 2002; 360: 503-504.

3. FAO Fisheries \& Aquaculture - Risks of fishing [Internet]. Available from: http://www.fao.org/fishery/topic/12383/en [cited 2015 Dec 29].

4. Fishery statistics in detail - Statistics Explained [Internet]. Available from: http://ec.europa.eu/eurostat/statistics-explained/index. php/Fishery_statistics_in_detail [cited 2015 Dec 29].

5. Jaremin B, Kotulak E. Mortality in the Polish small-scale fishing industry. Occup Med Oxf Engl 2004; 54: 258-260.

6. Matheson C, Morrison S, Murphy E, Lawrie T, Ritchie L, Bond C. The health of fishermen in the catching sector of the fishing industry: a gap analysis. Occup Med Oxf Engl 2001; 51: 305-311.

7. Lawrie T, Matheson C, Ritchie L, Murphy E, Bond C. The health and lifestyle of Scottish fishermen: a need for health promotion. Health Educ Res 2004; 19: 373-379.

8. Safety-in-the-Danish-fishing-industry.pdf [Internet]. Available from: http://teamarbejdsliv.dk/wp-content/uploads/Safety-in-theDanish-fishing-industry.pdf [cited 2015 Dec 29].

9. Frantzeskou E, Kastania AN, Riza E, Jensen OC, Linos A. Risk factors for fishermen's health and safety in Greece. Int Marit Health 2012; 63: 155-161.

10. Georgousopoulou EN, Panagiotakos DB, Pitsavos C, Stefanadis C, ATTICA Study Group. Assessment of diet quality improves the classification ability of cardiovascular risk score in predicting future events: the 10-year follow-up of the ATTICA study (2002-2012). Eur J Prev Cardiol 2015; 22: 1488-1498.

11. European Commision Public Health Programme. Development of Public Health Indicators for Reporting Environmental/Occupational
Risks Related to Agriculture and Fisheries (DIRERAF) [Internet]. 2004 Available from: http://euprojects.org/direraf.com/theproject.php.htm [cited 2015 Dec 29].

12. Martinez-Gonzalez MA, Fernandez-Jarne E, Serrano-Martinez M, Wright M, Gomez-Gracia E. Development of a short dietary intake questionnaire for the quantitative estimation of adherence to a cardioprotective Mediterranean diet. Eur J Clin Nutr 2004; 58: 1550-1552.

13. Panagiotakos DB, Pitsavos C, Arvaniti F, Stefanadis C. Adherence to the Mediterranean food pattern predicts the prevalence of hypertension, hypercholesterolemia, diabetes and obesity, among healthy adults; the accuracy of the MedDietScore. Prev Med 2007; 44: 335-340.

14. Marco Jordán L, Emparanza Knorr JL, Sarrasqueta Eizaguirre C, Gastesi Caballero C, Zubillaga Garmendía G. [Respiratory disorders among fishermen]. Med Clínica 1995; 105: 211-215.

15. Neitzel RL, Berna BE, Seixas NS. Noise exposures aboard catcher/processor fishing vessels. Am J Ind Med 2006; 49: 624-633.

16. Mamplekou E, Bountziouka V, Psaltopoulou T et al. Urban environment, physical inactivity and unhealthy dietary habits correlate to depression among elderly living in eastern Mediterranean islands : the MEDIS (MEDiterranean Islands Elderly) study. J Nutr Health Aging 2010; 14: 449-455.

17. Percin F, Akyol O, Davas A, Saygi H. Occupational health of Turkish Aegean small-scale fishermen. Occur Med (Lond) 2012; 62: 148-151.

18. Antonogeorgos G, Panagiotakos D, Pitsavos C et al. Understanding the role of depression and anxiety on cardiovascular disease risk, using structural equation modeling; the mediating effect of the Mediterranean diet and physical activity: the ATTICA study. Ann Epidemiol 2012; 22: 630-637. 